\title{
DESIGN OF THE EXPERIMENTAL EQUIPMENT TO IMPROVE STAMPING WITH ELASTIC MEDIA
}

\author{
VILCANS, J[anis]; TORIMS, T[oms] \& AVISANE, A[nita]
}

\begin{abstract}
This paper outlines experimental analysis of rubberpad forming, combined with part of the PhD research into sheet metal forming with elastic media. This paper covers analysis of the literature on rubber-pad forming and exploration of experimental equipment building. This equipment can be used to test dependence on surface roughness friction in the technological process of stamping with elastic media. By identifying the dependence on friction between the sheet material, base plate and elastic area surfaces, it is possible to precisely calculate the minimum size of the technological bridge. Further research is therefore required in order to enhance the efficiency of rubber pad forming technology.

Keywords: elastic media, experimental equipment, thin sheet metal
\end{abstract}

\section{INTRODUCTION}

Although a large number of machine components are made from sheet metal with a thickness of up to $2 \mathrm{~mm}$, production of such parts is not economically viable in traditional dies, which are designed for mass production.

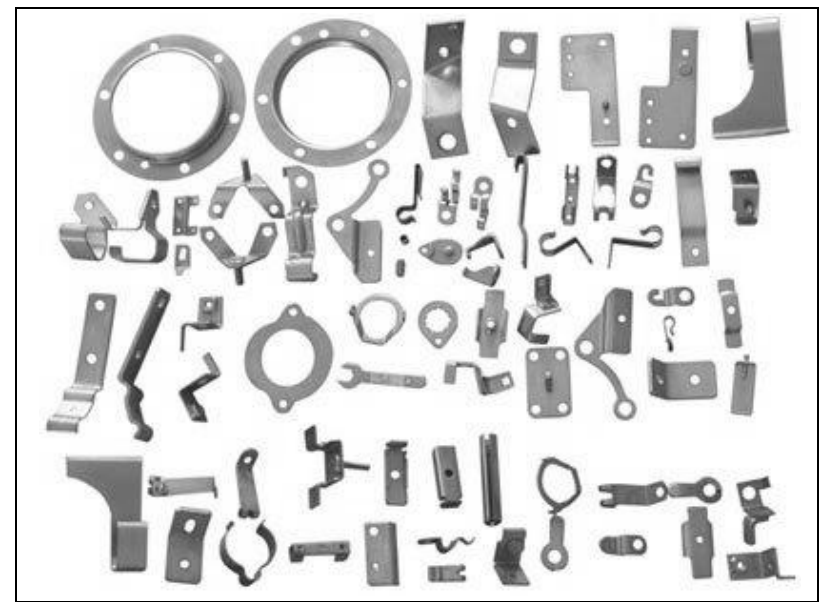

Fig. 1. Some examples of sheet metal parts

(source: http://www.cableglandsindia.com)

Sheet metal parts are deformed by the stamping process. The technological costs prove too high. Compared with traditional technological methods of manufacturing details from sheet material, forming with elastic media appears to be the optimal choice. This is especially true if the production run is less than 10,000 pieces. [3]

However, the drawback of this method is the relatively high amount of technological wastage, especially when cutting or stretching operations are performed. The relatively large technological bridges are justified by the need for them to withstand the force applied and remain in place. [5], [6], [7]

Further research is therefore required to improve the rubber-pad forming technology for industrial manufacturing, including $\mathrm{PhD}$ research into "Metal stamping in a flexible environment and process exploration" conducted at Riga Technical University, whose audit results are compiled in this article. This will help determine and experimentally confirm the minimum necessary size of the technological bridges. Consequently, it should be possible to reduce technological wastage, as well as consumption of the actual sheet material. [5, 6]

\section{OVERVIEW OF RUBBER-PAD FORMING LITERATURE}

The size of the technological bridges is relatively large because they have to withstand the working force and remain in place. The following parameters are normally used when calculating the dimensions of these bridges: a) geometry; b) force required to cut details; and c) actual holding force or friction. Naturally, the friction is directly related to the surface properties and roughness parameters.

In the case of forming with elastic media, the friction phenomena can be observed between the elastic area and the sheet material, as well as the base die plate.

Although preliminary research aiming to justify this technology has been successfully completed, it needs to be enhanced in order to obtain initial results that can be scientifically proven.

In the past, traditional rubber was widely used as a flexible environment. Nowadays it has been substituted in most cases by polyurethane, which has superior physical properties and can better withstand cyclic workloads. Therefore, although recent publications on stamping technology sometimes mention the existence of the aforementioned technology, they rarely provide any basic drawing samples. They also fail to provide any detailed description or mathematical model for metal stamping with elastic media. [5], [6], [7]

Our work aims to obtain an empirical model, which will recommend various degrees of roughness for the stamping dies, when stamping with flexible environment technology. In addition, the experimental results will be 
compared with the mathematically modeled analytical results. These issues are studied in depth. [7]

\section{COURSE OF RESEARCH}

Following analysis of the literature, the hypothesis was that surface roughness is a highly significant factor for clamping force and the quantity of wastage. [1], [2], [3], [4], [7] During the PhD research, there was call to explore the forces at work in the rubber pad stamping process and how the possibility of changing these forces depends on surface roughness parameters.

Research objects were chosen which are closely connected with the blank. It is underlying die, stamping material and elastic media-polyurethane. Inquire into interaction of underlying die, stamping material and elastic media (polyurethane) is possible get information about how to use stamping with elastic media technology with smaller technological wastages. Achieved results will be used to get information about the most efficient surface friction and at which surface roughness can get maximal friction. From experiment is possible get connections, from which is possible made mathematic model which shows processes that happen by using stamping with elastic media technology.

To obtain the relationship between the forces and surface roughness using rubber pad stamping technology. Need to make analysis of process which is acting from both sides of blank. It means how acting blank surface with elastic media and blank surface with underlying pad surface. Schematically it showed in "Fig. 2"

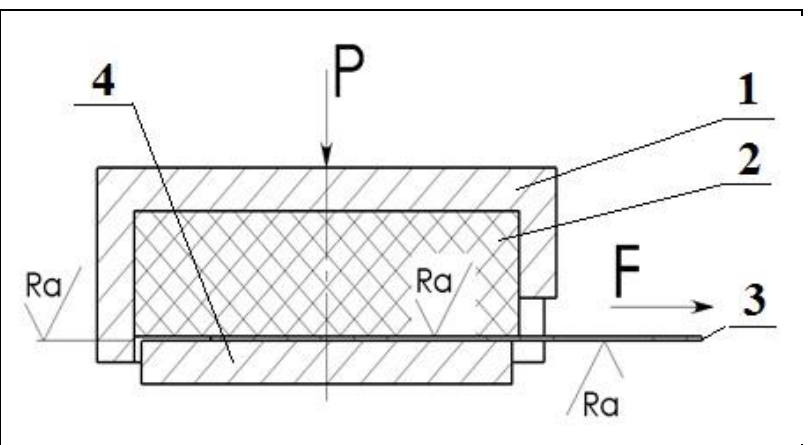

Fig. 2. Stamping with elastic media scheme. 1. Container of elastic media 2. Elastic media-polyurethane 3. Blank 4. Underlying pad

Research of these processes need to be done in one system because these processes interacting form each other. Puling force (F) "Fig. 2" closely interacts with clamping force $(\mathrm{P})$ "Fig. 2". If increase clamp force (P) increasing force $(\mathrm{F})$ which is needed for pulling out blank sample "Fig. 2 position 3". This phenomenon happens because increasing friction from both sides of blank. It means friction between blank surface with elastic media - polyurethane and blank surface with underlying pad surface.

As the aim of the experiment was launched to find out which is minimal clamping force $(\mathrm{P})$ at which is needed maximal puling force $(\mathrm{F})$. Or in other words, what is the interaction model of puling force and pressure force and interrelationships.
On the basis of the description of the technology was selected the parameters that are most affected by the system and discarded the parameters of the system is affected the least. Schematically showed in "Tab. 1". [3], [7]

\begin{tabular}{|l|l|l|}
\hline Input factors & & Output factors \\
\cline { 1 - 1 } Pressure force (P) & $\Rightarrow$ & Pull-out force (F) \\
Polyurethane elasticity & $\Rightarrow$ & \\
Underlying properties & $\Rightarrow$ & \\
Blank properties & $\Rightarrow$ & \\
\hline
\end{tabular}

\subsection{Experimental equipment}

Was made analysis for available equipment in Riga Technical University and conclusion was that necessary equipment needed to build by self.

The equipment for experiments was required to provide technological system. For experimental equipment was required to provide, that you can determine what the compression force on the material is and what friction processes is between the underlying pad surface and the blank and the processes in friction between flexible environment and blank. Need to materialize table 1conditions. Using the available material and technical facilities need to build experiment equipment. Must met not only the conditions defined in the system but also to ensure the easy way how to change blank and replacement blank must stay on the same place as previously. Main construction must be build from two halves. From one side of blank must be container with elastic media, in my case polyurethane, fixed half. And from other side all other construction, movable half. Movable half must to ensure the clamping on the machine on movable desk and all movable part elements must move together.

Setting up experimental equipment need to solve the following problems:

1. Choose a device on which to perform experiments. (it can be press or tensile stress tester )

2. Choose a compression - clamping force meter reading (compression dynamometer)

3. Choose pulling force meter reading (tensile dynamometers)

4. Chose a blank material and dimensions. Workpiece material and dimensions should be such that it does not exceed the tensile strength in tension dynamometer for measuring the field boundary.

5. Must be made flexible environment container according to the selected device (1). According to the clamping force dynamometer (2).Container wall must be milled thru to ensure the exemption according to the selected workpiece blank (4). Predict the approximate depth of the exemption 
depending on the elastic deformation of the flexible environment.

6. Must be made changeable dies according to the planned experiments need to make a die with a replaceable base surface to be able to easy change the surface roughness parameters.

7. Must be made clamp for the blank (4). Clamp must connect a tensile force measuring system (3). Clamp provides compressive strength greater than the strength the blanks border tension.

8. Must be made of fastening elements which link the main die (6) with a tension dynamometer (3) so as to be able to stretch. It would provide a fixed support, and the sufficient strength, which is greater than the blank (4) strength.

9. Must be made tension mechanism in the middle of tensile dynamometers (3) and fastening element (8).

How you see more of components what must be made are very closely dependent from each other.

Given the above considerations was decided to use press or tensile stress tester Zwick/RoelZ150 "Fig. 3"

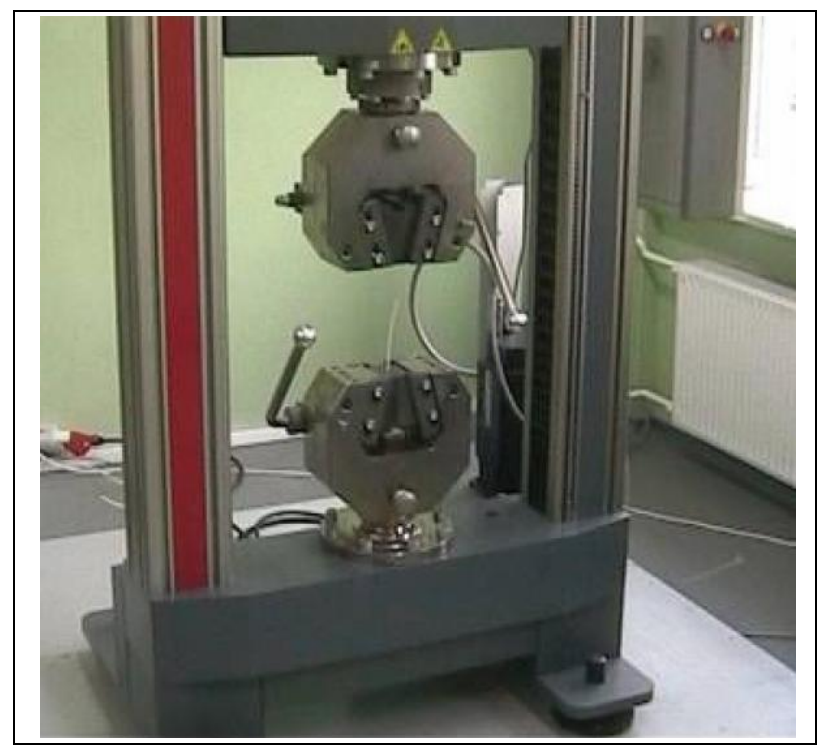

Fig. 3. Press or tensile stress tester Zwick/RoelZ150

Given the above considerations was decided to use digital dynamometer U2A tensile loading to a maximum of up to $100 \mathrm{~kg}$. "Fig. 4"

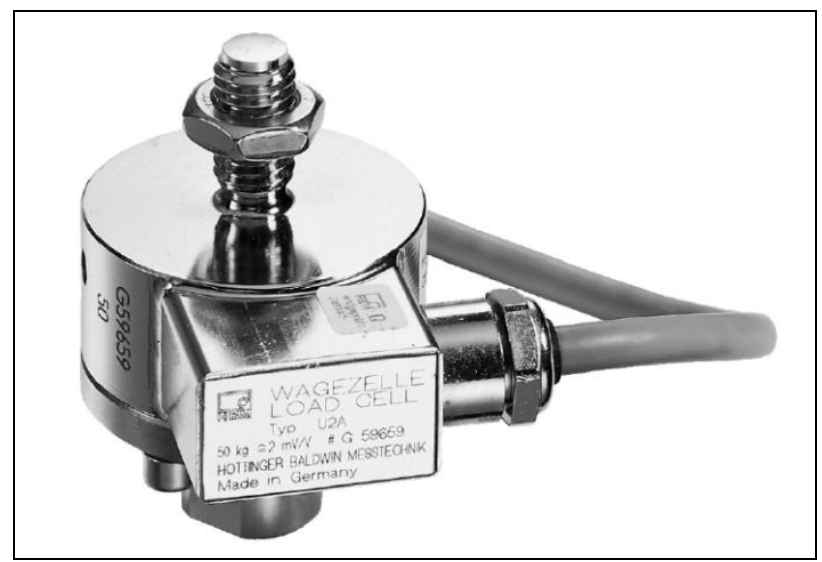

Fig. 4. Digital dynamometer U2A
Knowing that the blank ultimate strength (Pmaxdin) must not exceed $100 \mathrm{~kg}$ of strength, then is possible select the blanks plate cross-sectional dimensions. For blank samples were selected material, which is widely used in stamping process and is freely available at outlets EN10130 DC01 brand sheet metal and the blanks samples with dimensions $0.5 \times 4.5 \times 150 \mathrm{~mm}$.

Selected blank samples were experimentally tested to tensile resistance with testing machine Zwick/Roel Z150 and all the results were very similar. Tensile test of the blank samples with a cross section $0.5 \times 4.5 \mathrm{~mm}$ resistance was $\approx 90 \mathrm{~kg}$ load. "Fig. 5"

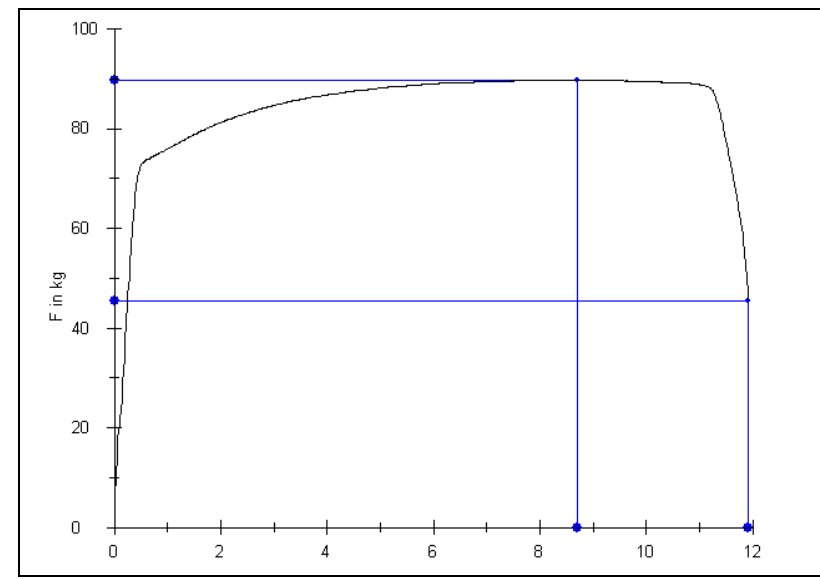

Fig. 5. Tensile diagram for blank sample $0.5 \times 4.5 \mathrm{~mm}$ cross section and material EN10130 DC01

Using above considerations was built experimental equipment which satisfies the required conditions. "Fig. $6 "$

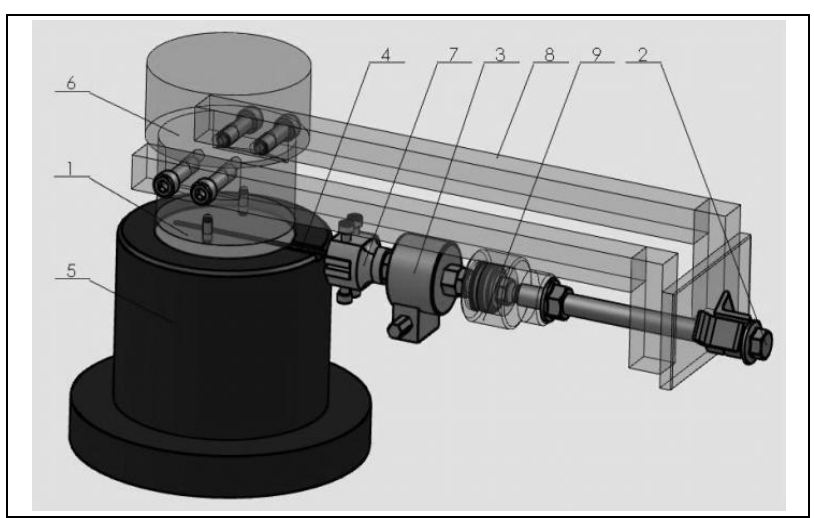

Fig. 6. Experimental equipment where 1-exchangeable die surface, 2 tensioning screw, 3-tensile dynamometers, 4 - blank sample, 5-elastic environment container with a flexible environment-polyurethane, 6-die holder, 7 - clamp, 8 - support.

The experimental equipment was manufactured and the first tests were carried out.

\subsection{Experiment inputs}

Looking at the literature [1], [2], [3], [4], [7] was concluded that the greatest effect on the output factor extraction force (F) "Fig. 2" is given by the input factor compressive force (P) "Fig. 2". During the experiment force $(\mathrm{P})$ initially set up as a constant, but when starts force $(F)$ measurements, it will also change force $(P)$ value. The reason is: pulling out of the workpiece will change the contact area between the blank and flexible 
environment, as well as between the workpiece and the basic surface.

The first tests were done in relationship with table 1 data. To be constant for each experiment will be incorporated within the framework of:

- Die surface roughness parameters

- Blank sample surface roughness parameters

- Blank sample material properties

- Elastic media properties

\section{RESULTS OF FIRST TESTS}

The first experiments were carried out «Fig. 7". and the first results were obtained. Where ensiled several blank samples and established limits of the compressive forces. Where tensiled three blank samples on three main die surfaces, which were previously treated with different treatment technologies turning, milling and grinding.

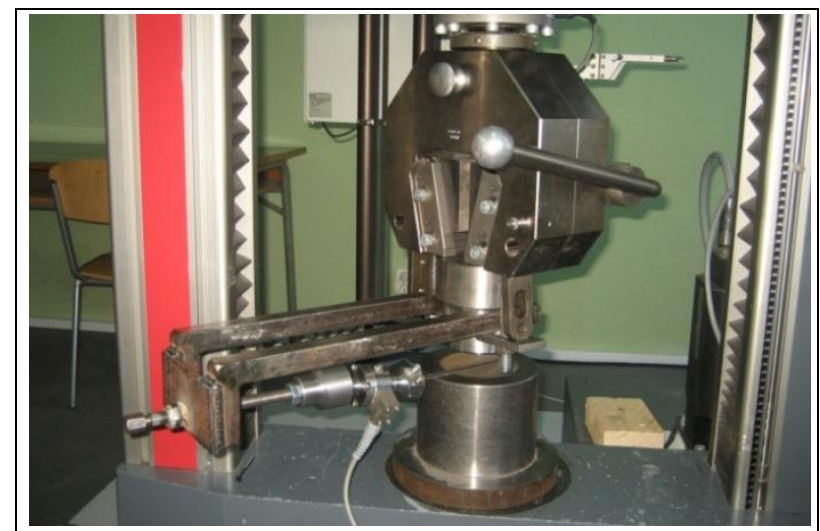

Fig. 7. Experimental equipment mounted on press or tensile stress tester Zwick/RoelZ150

During the experiment the selected workpiece were determined empirically researched significance $(\mathrm{P})$ "Fig. 2"values 300 - $700 \mathrm{~kg}$. Squeezing blank with less force than 300 slips and squeezing the workpiece with the force of more than $700 \mathrm{~kg}$ it certainly is clamped in position.

Due to the fact that during the experiment the two dynamometers could not read from one device to the experiments was set at a constant compression force $(\mathrm{P})$ and pulled out the blank sample. It was concluded; that, from this experiment we can obtain information only on a given compressive force $(\mathrm{P})$ is the blanks slip or is certainly clamped in position. And when the blank sample is clamped in position from the experimental data is obtained the tensile fracture diagram.

\section{FUTURE WORK}

Next step will be finding solutions how to read the two synchronized force values ( $P$ and $F$ ). In this way more accurately reflect what is happening in the study system.

Future research will be focused on empirical system model. It can be developed as a result of recommendation of roughness choice of stamping die using stamping with flexible environment technology. In addition, the experimental results will be compared with the analytical results obtained mathematically modeled.

\section{CONCLUSION}

However, the drawback of stamping with elastic media is the relatively high quantity of technological wastage, owing to the technological bridge size. Research is therefore required to improve the rubber pad stamping technology for industrial manufacturing. Experimental research is needed to determine the significance of the underlying surface roughness and to improve the surface characteristics.

During the first experiment established limits of the compressive forces and was founded weak point of construction. Need to find solutions how to read the two synchronized force values

The work here will provide a basis for future experiments, and make it possible to draw an empirical model that can be developed as a result of recommendation of roughness choice of stamping die using stamping with flexible environment technology. In addition, the experimental results will be compared with the analytical results obtained mathematically modeled.

\section{ACKNOWLEDGEMENTS}

This work has been supported by the European Social Fund under Project Nr. 2009 / 0201 / 1DP / 1. 1. 1. 2. 0 / 09 / APIA / VIAA / 112 "Nanotechnological research of the mechanical element surface and internal structure in mechanical engineering".

\section{REFERENCES}

[1] Bingato, T., (2010), "Development of multistep inverse finite element method and its application in formability prediction of multistage sheet metal forming", Journal of Manufacturing Science and Engineering, Vol. 132 (August 2010), pp 041013-1 041013-9, ISSN 1087-1357

[2] Guo, Y.Q., (2004), "An efficient pseudo-inverse approach for damage modelling the sheet forming process", Journal of Materials Processing Technology (2004), ISSN: 09240136

[3] Hisaki W., (2000). Flexible methods for punching a thin metal sheet using a urethane sheet. In Proceedings of the $33 \mathrm{rd}$ International MATADOR Conference, pp 413-418, ISBN 9781852333232, London

[4] Kalpakjian, S., (2006) Manufacturing Engineering and Technology, Publisher: Pearson Prentice Hall; 5th edition, ISBN13: 978-0131976399, Saddle River, NJ

[5] Vilcans. J.; Torims. T.; Zarins. M. \& Ratkus. A. (2011). Experimental Analysis of the Rubber Pad Forming. Annals of DAAAM for 2011 \& Proceedings of the 22nd International DAAAM Symposium. 23-26th November 2011. Vienna. Austria. Volume 22. No. 1. ISSN 1726-9679. ISBN 978-3-901509-83-4. Katalinic. B. (Ed.), pp. 0613-0614. Published by DAAAM International Vienna. Vienna

[6] Vilcans, J. \& Torims, T. (2010). Thin Sheet Metal Stamping with Elastic Media, Annals of DAAAM for 2010 \& Proceedings of the 21st International DAAAM Symposium, 20-23rd October 2010, Zadar, Croatia, ISSN 1726-9679, ISBN 978-3-901509-73-5, Katalinic, B. (Ed.), pp. 0849-0850, Published by DAAAM International Vienna, Vienna

[7] Khodyrev, A. (1973) Design, construction and operation with polyurethane stamps (Штамповка полиуретаном деталей из листовых материалов), University of Perm 